\title{
ON THE EXISTENCE OF ORDINARY TRIANGLES
}

\author{
RADOSLAV FULEK, HOSSEIN NASSAJIAN MOJARRAD, MÁRTON NASZÓDI, \\ JÓZSEF SOLYMOSI, SEBASTIAN U. STICH, AND MAY SZEDLÁK
}

\begin{abstract}
Let $P$ be a finite point set in the plane. A $c$-ordinary triangle in $P$ is a subset of $P$ consisting of three non-collinear points such that each of the three lines determined by the three points contains at most $c$ points of $P$. Motivated by a question of Erdős, and answering a question of de Zeeuw, we prove that there exists a constant $c>0$ such that $P$ contains a $c$-ordinary triangle, provided that $P$ is not contained in the union of two lines. Furthermore, the number of $c$-ordinary triangles in $P$ is $\Omega(|P|)$.
\end{abstract}

\section{INTRODUCTION}

In 1893, Sylvester [Syl93] asked whether, for any finite set of non-collinear points on the Euclidean plane, there exists a line incident with exactly two points. The positive answer to this question, now known as the Sylvester-Gallai theorem, was first obtained almost half a century later in 1941 by Melchior [Mel41] as a consequence of the positive answer to an analogous question in the projective dual. Erdös [EBW $\left.{ }^{+} 43\right]$, unaware of these developments, posed the same problem in 1943, and it was solved by Gallai in 1944. For more on the history of this and related problems, see GT13.

Given a finite set of points $P$ on the Euclidean plane, a line $\ell \subset \mathbb{R}^{2}$ is determined by $P$ if $\ell$ contains at least two points of $P$. We say that $\ell$ is an ordinary line, if $\ell$ contains exactly two points of $P$. Erdös [Erd84] considered the problem of finding an ordinary triangle, that is, three ordinary lines determined by three points of a finite planar point set. See [BM90] for details on the origin of this problem. Motivated by this problem, and with an application in studying ordinary conics BVdZ16, de Zeeuw asked a related question at the 13th Gremo's Workshop on Open Problems (GWOP 2015, Feldis, Switzerland), which we describe below.

Definition 1.1 (c-ordinary triangle). Let $c$ be a natural number and let $P$ be a point set in the plane. A c-ordinary triangle in $P$ is a subset of $P$ consisting of three non-collinear points such that each of the three lines determined by the three points contains at most $c$ points of $P$.

It is easy to see that in order to be able to find a $c$-ordinary triangle for large $n$, we have to assume that $P$ is not contained in the union of two lines. Under this restriction one might suspect that there is a 2-ordinary triangle in $P$. However, this is not true as shown by Böröczky's construction GT13, Figure 4,5,6]. The following simple example also shows this. Let $P_{1}$ be a set of points that are not all collinear and let $\ell$ be some line. For each line $\ell_{1}$ determined by the point set $P_{1}$, we add the point at the intersection of $\ell$ and $\ell_{1}$. Let us denote this new set of points by $P_{2}$. All points of $P_{2}$ are collinear, hence a 2-ordinary

2010 Mathematics Subject Classification. 52C30.

Key words and phrases. Dirac-Motzkin Conjecture, incidences, ordinary lines, ordinary triangle, planar point set. 
triangle must contain two points from $P_{1}$. However, by construction every line determined by $P_{1}$ contains a point of $P_{2}$. Hence there are no 2-ordinary triangles in this point set.

De Zeeuw asked whether a $c$-ordinary triangle can be found in $P$. The aim of this manuscript is to give a positive answer to this question.

Theorem 1.2. There is a natural number $c$ such that the following holds. Assume $P$ is a finite set of points on the Euclidean plane not contained in the union of two lines. Then $P$ contains a c-ordinary triangle, that is three non-collinear points such that each of the three lines determined by these three points contains at most c points of $P$. Moreover, the number of c-ordinary triangles in $P$ is $\Omega(|P|)$.

Remark 1.3. The constant in the theorem above can be chosen as $c=12000$.

We see no reason to believe that this is the best constant. Moreover, it remains open if the number of $c$-ordinary triangles in $P$ is superlinear (possibly even quadratic) in $|P|$.

\section{TOOLS}

To prove Theorem 1.2, we need the following lemmas. The first one is a corollary of the Szemerédi-Trotter Theorem.

Lemma 2.1. [ST83, PRTT06] Let $k, n \geq 2$ be natural numbers, $P$ a set of $n$ points in the plane, and let $f(k)$ denote the number of lines in the plane containing at least $k$ points of $P$. Then

$$
f(k) \leq\left\{\begin{array}{l}
c^{\prime} \frac{n^{2}}{k^{3}}, \text { if } k \leq \sqrt{n} \\
c^{\prime} \frac{n}{k}, \text { if } k>\sqrt{n}
\end{array}\right.
$$

for a universal constant $c^{\prime}>0$. In fact, we may take $c^{\prime}=125$.

Proof. Clearly, the claimed bound holds for $k=2,3$, since $f(2) \leq\left(\begin{array}{l}n \\ 2\end{array}\right)$ and $f(3) \leq\left(\begin{array}{l}n \\ 2\end{array}\right) /\left(\begin{array}{l}3 \\ 2\end{array}\right)$. To prove the statement for $k>3$, we rely on the following result by Pach, Radoičić, Tardos and Tóth [PRTT06, Corollary 5.1]: for any given $n$ points and $m$ lines on the Euclidean plane, the number of incidences between them is at most $2.5 m^{2 / 3} n^{2 / 3}+m+n$. Let $m=f(k)$ denote the number of lines containing at least $k$ points of $P$. Observe that the number of point-line incidences are thus at least $m k$. Hence, $m k \leq 2.5 m^{2 / 3} n^{2 / 3}+m+n$.

First, consider the case $m \geq n$. Observe that for $k>3$, we have $m k / 2 \leq m(k-2) \leq$ $2.5 m^{2 / 3} n^{2 / 3}$. It follows that $m \leq 125 \frac{n^{2}}{k^{3}}$, and specifically, $m \leq 125 \frac{n}{k}$ if $k>\sqrt{n}$.

Next, consider the case $m<n$. We have $m k \leq 2.5 m^{2 / 3} n^{2 / 3}+m+n \leq 2.5 m^{2 / 3} n^{2 / 3}+2.5 n$, and therefore $m k \leq \max \left\{5 m^{2 / 3} n^{2 / 3}, 5 n\right\}$. Hence, $m \leq \max \left\{125 \frac{n^{2}}{k^{3}}, 5 \frac{n}{k}\right\}$. For $k \leq 5 \sqrt{n}$, the maximum is attained at the first term, whereas for $k>\sqrt{n}$, we trivially have $\frac{n^{2}}{k^{3}}<\frac{n}{k}$, establishing the claim for $c^{\prime}=125$.

The following Turán-type lemma (related to Mantel's theorem) from extremal graph theory provides a lower bound for the number of triangles (subgraphs isomorphic to $K_{3}$ ) in a graph. It can be found with a proof as Problem 10.33 in [Lov07.

Lemma 2.2. Consider a graph $G=(V(G), E(G))$ with $|V(G)|=n$ and $|E(G)|=m$. Let $t_{3}(G)$ denote the number of triangles in $G$. Then we have

$$
t_{3}(G) \geq \frac{m}{3 n}\left(4 m-n^{2}\right) .
$$




\section{Proof of Theorem 1.2}

In this section, we prove Theorem 1.2. Our proof is closely related to the standard proof of Beck's Theorem, where the number of pairs of points on medium-rich lines is bounded using the Szemerédi-Trotter theorem, and then it is concluded that either there is a very rich line, or there are many pairs of points on poor lines, see the proof of Theorem 18.8 in Juk11.

The constant $c$ will be chosen at the end of the proof. Assume $P$ is a set of $n \geq c$ points in the plane and let $\mathcal{L}=\left\{L_{1}, L_{2}, \ldots, L_{m}\right\}$ denote the set of lines determined by $P$. Define $l_{i}=\left|L_{i} \cap P\right|$, for $i=1,2, \ldots, m$.

Set $\alpha=\frac{4}{c+1}$. We split the proof into two cases:

(i) There is a line $L_{i} \in \mathcal{L}$ such that $l_{i}>\alpha n$;

(ii) For all $i=1,2, \ldots, m$ we have $l_{i} \leq \alpha n$.

Consider the first case. Since the point set $P \backslash L_{i}$ is non-collinear by the assumption, by applying the Sylvester-Gallai theorem, we can find an ordinary line $L \in \mathcal{L}$ for $P \backslash L_{i}$, i.e. $L$ contains exactly two points $q, r \in P \backslash L_{i}$. Note that $L$ may contain at most one point of $P \cap L_{i}$. Next, we show that there are many points on $L_{i}$ which together with $q, r$ form $c$-ordinary triangles. For this, we define the set $P_{q} \subset P$ as

$$
P_{q}=\left\{p \in L_{i} \cap P:|\overline{p q} \cap P|>c\right\},
$$

where $\overline{p q}$ denotes the line passing through $p, q$. We define $P_{r}$ in a similar way. Note that for any point $p \in P_{q}$, the line $\overline{p q}$ contains at least $c-1$ points of $P \backslash\left(L_{i} \cup\{q\}\right)$, moreover, these sets of $c-1$ points are disjoint for different $p \in P_{q}$. So we get

$$
(c-1) \cdot\left|P_{q}\right| \leq n-l_{i},
$$

which implies that

$$
\left|P_{q}\right| \leq \frac{n-l_{i}}{c-1}<\frac{l_{i} / \alpha-l_{i}}{c-1}=\frac{(c+1) / 4-1}{c-1} \cdot l_{i}<\frac{l_{i}}{4} .
$$

Similarly, $\left|P_{r}\right|<\frac{l_{i}}{4}$. So there are at least $\frac{l_{i}}{2}$ points $s \in P \cap L_{i}$ such that $s \notin P_{q} \cup P_{r}$. Furthermore, $s, q, r$ are non-collinear. This implies that the lines $\overline{s q}, \overline{s r}$ contain at most $c$ points of $P$. Therefore every triangle determined by $s, q, r$, where $s \notin P_{q} \cup P_{r}$, is a $c$-ordinary triangle for $P$. The number of these triangles is at least $\frac{l_{i}}{2}>\frac{\alpha n}{2}=\frac{2 n}{c+1}$, completing the proof of case (i). Note that, so far, $c$ may be chosen as any integer greater than 2 .

Next, we consider case (ii). So we assume that no line of $\mathcal{L}$ contains more than $\alpha$ points of $P$. First we bound $\sum_{c<l_{i} \leq \alpha n}\left(\begin{array}{c}l_{i} \\ 2\end{array}\right)$ from above. With the notation of Lemma 2.1, we have

$$
\begin{aligned}
\sum_{i: c<l_{i} \leq \sqrt{n}}\left(\begin{array}{c}
l_{i} \\
2
\end{array}\right) & \leq \sum_{j=\lfloor\log (c+1)\rfloor}^{\lceil\log \sqrt{n}\rceil} \sum_{i: 2^{j} \leq l_{i} \leq 2^{j+1}}\left(\begin{array}{c}
l_{i} \\
2
\end{array}\right) \leq \sum_{j=\lfloor\log (c+1)\rfloor}^{\lceil\log \sqrt{n}\rceil} f\left(2^{j}\right)\left(\begin{array}{c}
2^{j+1} \\
2
\end{array}\right) \\
& \leq \sum_{j=\lfloor\log (c+1)\rfloor}^{*} c^{\prime} \frac{n^{2}}{2^{3 j}}\left(\begin{array}{c}
2^{j+1} \\
2
\end{array}\right) \leq \sum_{j=\lfloor\log (c+1)\rfloor}^{\lceil\log \sqrt{n}\rceil} c^{\prime} \frac{n^{2}}{2^{j-1}} \\
& \leq \sum_{j=\lfloor\log (c+1)\rfloor}^{\infty} c^{\prime} \frac{n^{2}}{2^{j-1}} \leq \frac{8 c^{\prime} n^{2}}{c+1}
\end{aligned}
$$


where logarithms are base 2, and the inequality with star follows from Lemma 2.1.

On the other hand, by the same lemma, we have

$$
\begin{aligned}
\sum_{i: \sqrt{n}<l_{i} \leq \alpha n}\left(\begin{array}{c}
l_{i} \\
2
\end{array}\right) & \leq \sum_{j=0}^{\lceil\log (\alpha \sqrt{n})\rceil-1} \sum_{2^{j} \sqrt{n}<l_{i} \leq 2^{j+1} \sqrt{n}}\left(\begin{array}{c}
l_{i} \\
2
\end{array}\right) \leq \sum_{j=0}^{\lceil\log (\alpha \sqrt{n})\rceil-1} f\left(2^{j} \sqrt{n}\right)\left(\begin{array}{c}
2^{j+1} \sqrt{n} \\
2
\end{array}\right) \\
& \leq \sum_{j=0}^{*} c^{\prime} \frac{n}{2^{j} \sqrt{n}}\left(\begin{array}{c}
2^{j+1} \sqrt{n} \\
2
\end{array}\right) \\
& \leq \sum_{j=0}^{\lceil\log (\alpha \sqrt{n})\rceil-1} c^{\prime} n^{3 / 2} 2^{j+1} \leq 4 c^{\prime} n^{3 / 2} \cdot \alpha \sqrt{n}=\frac{16 c^{\prime} n^{2}}{c+1} .
\end{aligned}
$$

As a result, we obtain

$$
\sum_{i: c<l_{i} \leq \alpha n}\left(\begin{array}{l}
l_{i} \\
2
\end{array}\right)=\sum_{i: c<l_{i} \leq \sqrt{n}}\left(\begin{array}{c}
l_{i} \\
2
\end{array}\right)+\sum_{i: \sqrt{n}<l_{i} \leq \alpha n}\left(\begin{array}{l}
l_{i} \\
2
\end{array}\right) \leq \frac{24 c^{\prime} n^{2}}{c+1} .
$$

Let $G$ be the graph with vertex set $V(G)=P$, such that two points $p, p^{\prime} \in P$ are adjacent in $G$ if the line $\overline{p p^{\prime}}$ spanned by $p, p^{\prime}$ satisfies $\left|\overline{p p^{\prime}} \cap P\right| \leq c$.

By the following identity

$$
\sum_{i: 2 \leq l_{i} \leq \alpha n}\left(\begin{array}{l}
l_{i} \\
2
\end{array}\right)=\left(\begin{array}{l}
n \\
2
\end{array}\right),
$$

we obtain for the number of edges of $G$,

$$
|E(G)|=\sum_{i: 2 \leq l_{i} \leq c}\left(\begin{array}{l}
l_{i} \\
2
\end{array}\right) \geq\left(\begin{array}{l}
n \\
2
\end{array}\right)-\frac{24 c^{\prime} n^{2}}{c+1} .
$$

Now we choose $c$ large enough such that

$$
4\left(\left(\begin{array}{l}
n \\
2
\end{array}\right)-\frac{24 c^{\prime} n^{2}}{c+1}\right)-n^{2}=\Omega\left(n^{2}\right) .
$$

Combining it with (1) yields

$$
4|E(G)|-n^{2}=4\left(\sum_{i: 2 \leq l_{i} \leq c}\left(\begin{array}{c}
l_{i} \\
2
\end{array}\right)\right)-n^{2}=\Omega\left(n^{2}\right) .
$$

Therefore, by Lemma 2.2 we have

$$
t_{3}(G) \geq \frac{|E(G)|}{3 n}\left(4|E(G)|-n^{2}\right)=\frac{\Omega\left(n^{2}\right)}{n} \cdot \Omega\left(n^{2}\right)=\Omega\left(n^{3}\right) .
$$

This implies that $G$ has $\Omega\left(n^{3}\right)$ triangles. Let $T$ be the set of those triangles in $G$ whose three vertices are non-collinear. It is easy to see that these triangles correspond to $c$-ordinary triangles in $P$.

Note that the number of triangles with collinear vertices is at most

$$
\sum_{i: 2 \leq l_{i} \leq c}\left(\begin{array}{l}
l_{i} \\
3
\end{array}\right) \leq \sum_{i: 2 \leq l_{i} \leq c}\left(\begin{array}{l}
c \\
3
\end{array}\right) \leq\left(\begin{array}{l}
n \\
2
\end{array}\right) \cdot\left(\begin{array}{l}
c \\
3
\end{array}\right)=O\left(n^{2}\right) .
$$


So we get

$$
|T|=\Omega\left(n^{3}\right)-O\left(n^{2}\right)=\Omega\left(n^{3}\right) .
$$

As a result, $P$ has $\Omega\left(n^{3}\right) c$-ordinary triangles, provided that $c$ satisfies (2).

Proof of Remark 1.3. Equation (2) yields that we may choose $c=96 c^{\prime}$, where $c^{\prime}$ is from Lemma 2.1.

\section{ACKNOWLEDGEMENTS}

We thank Emo Welzl for providing the venue, his GWOP workshop, where our research initially started. We also thank Frank de Zeeuw for his many remarks on earlier versions of the manuscript. We are grateful to both referees, whose comments made the presentation much cleaner.

R. Fulek was partially supported by the People Programme (Marie Curie Actions) of the European Union's Seventh Framework Programme (FP7/2007-2013) under REA grant agreement no [291734]. H. N. Mojarrad and M. Naszódi were members of János Pach's Chair of DCG at EPFL, Lausanne, supported by the Swiss National Science Foundation (SNSF) Grants 200020-162884 and 200021-165977. M. Naszódi was also partially supported by the National Research, Development and Innovation Office grant K119670, and by the János Bolyai Research Scholarship of the Hungarian Academy of Sciences. J. Solymosi was supported by NSERC, by ERC Advanced Research Grant no 267165 (DISCONV) and by the National Research, Development and Innovation Office (NKFIH) grant NK 104183. S. U. Stich acknowledges support from SNSF and grant "ARC 14/19-060" from the "Direction de la recherche scientifique - Communauté française de Belgique". M. Szedlák's research was supported by the SNSF Project 200021_150055/1.

\section{REFERENCES}

[BM90] Peter Borwein and William O. J. Moser, A survey of Sylvester's problem and its generalizations, Aequationes Mathematicae 40 (1990), no. 1, 111-135.

[BVdZ16] Thomas Boys, Claudiu Valculescu, and Frank de Zeeuw, On the Number of Ordinary Conics, SIAM Journal on Discrete Mathematics 30 (2016), no. 3, 1644-1659 (en).

$\left[\mathrm{EBW}^{+} 43\right]$ Paul Erdős, Richard Bellman, Hubert S. Wall, James Singer, and Victor Thébault, Problems for solution: 4065-4069, Amer. Math. Monthly 50 (1943), no. 1, 65-66.

[Erd84] Paul Erdős, Research problems, Period. Math. Hungar. 15 (1984), 101-103.

[GT13] Ben Green and Terence Tao, On sets defining few ordinary lines, Discrete \& Computational Geometry 50 (2013), no. 2, 409-468.

[Juk11] Stasys Jukna, Extremal combinatorics, second ed., Texts in Theoretical Computer Science. An EATCS Series, Springer, Heidelberg, 2011, With applications in computer science. MR 2865719

[Lov07] László Lovász, Combinatorial problems and exercises, second ed., AMS Chelsea Publishing, Providence, RI, 2007. MR 2321240

[Mel41] Eberhard Melchior, Über Vielseite der projektiven Ebene, Deutsche Mathematik 5 (1941), 461475.

[PRTT06] János Pach, Radoš Radoičić, Gábor Tardos, and Géza Tóth, Improving the crossing lemma by finding more crossings in sparse graphs, Discrete \& Computational Geometry 36 (2006), no. 4, $527-552$.

[ST83] Endre Szemerédi and William T. Trotter, Jr., Extremal problems in discrete geometry, Combinatorica 3 (1983), no. 3-4, 381-392.

[Syl93] James Joseph Sylvester, Mathematical question 11851, Educational Times 59 (1893), no. 98, 256. 